\title{
A Two-sided Matching Decision-making Approach based on PROMETHEE Under the Probabilistic Linguistic Environment
}

\author{
Xiang Jia ( $\sim$ 1932328534@qq.com ) \\ Hunan University of Technology https://orcid.org/0000-0002-5002-3660 \\ Yingming Wang \\ Fuzhou University
}

\section{Research Article}

Keywords: Probabilistic linguistic term sets, Two-sided matching, Optimal model, Matching aspiration

Posted Date: September 27th, 2021

DOl: https://doi.org/10.21203/rs.3.rs-724412/v1

License: (c) (1) This work is licensed under a Creative Commons Attribution 4.0 International License.

Read Full License 


\title{
A two-sided matching decision-making approach based on PROMETHEE under the probabilistic linguistic environment
}

\author{
Xiang Jia, Yingming Wang* \\ School of Economics and Management, Fuzhou University, Fuzhou 350108, China
}

\begin{abstract}
Matching problems in daily life can be effectively solved by two-sided matching decisionmaking (TSMDM) approaches. The involved matching intermediary is to match two sides of subjects. This paper proposes a TSMDM approach based on preference ranking organization method (PROMETHEE) under the probabilistic linguistic environment. The probabilistic linguistic evaluations are firstly normalized and transformed to the benefit types. Then, the preference degrees of a subject over other subjects from the same side are obtained by using six types of preference function. Afterwards, groups of preference degrees of a subject are aggregated to the preference indexes by considering the weights of criteria. Hereafter, the preference degrees of a subject over other subjects from the same side are aggregated to the outgoing flow, while the preference degrees of other subjects from the same side over this subject are aggregated to the incoming flow. Furthermore, the net-flows, which is recognized as the satisfaction degrees are calculated by using outgoing flows to minus incoming flows. On the basis of this, the multi-objectives TSMDM model is built by considering the matching aspirations. A model with respect to the matching aspirations is built and solved by using the Lagrange function. The multi-objectives TSMDM model is further transformed to the single-objective model, the solution of which is the matching scheme. A matching problem related to the intelligent technology intermediary is solved to verify the effectiveness and the feasibility of the proposed approach.
\end{abstract}

Keywords: Probabilistic linguistic term sets; Two-sided matching; Optimal model; Matching aspiration

\footnotetext{
* Corresponding author. E-mail addresses: ymwang@fzu.edu.cn (Y.M. Wang).
} 


\section{Introduction}

Two-sided matching decision-making (TSMDM) problems involve three elements: two sides of subjects and an intermediary. The task of the intermediary is to match two sides of subjects one by one, where the subjects are discrete and finite. Gale and Shapley (1962) introduced the TSMDM problems by investigating the college admissions and stability of marriage. On the basis of this, we recount the TSMDM problems by the example of marriage. Suppose there are three females and four males are to be matched by a marriage intermediary, then three females construct a side while four males construct the other side. The evaluations of females given by males and that of males given by females are previously obtained by the marriage intermediary, the task of which is to match males and females according to these evaluations. Since a male can only match a female, all females are matched, three males are matched and a male is not matched obviously. To deal with the matching problems, the approach the intermediary utilized is called the TSMDM approach. Since Gale and Shapley (1962) proposed the deferred-acceptance algorithm, lots of TSMDM approaches are extracted and further employed to the practical problems, such as the matching for technological knowledge suppliers and demanders (Han et al. 2018; Liu and Li 2017), the matching for two-way referral system (Wang et al. 2018), the matching in smart environmental protection (Yue et al. 2019).

Evaluations are important information for subjects to measure the subjects from the other side. Fan et al. (2017) considered the condition where the subjects can only give their uncertain preference ordinal and further proposed a TSMDM method considering elation and disappointment of subjects. Liang et al. (2020) considered the incomplete preference ordinal environment and extracted a TSMDM approach, where the missing information is expressed by using the similarity measures. Considering the fuzziness of the subjects when giving their evaluations, Wang et al. (2016), Yu and $\mathrm{Xu}$ (2019), Yin and Li (2018) proposed several TSMDM approaches, where the evaluations are expressed as intuitionistic fuzzy sets. Yue et al. (2016) extended the TSMDM approach to accommodate the interval-valued intuitionistic fuzzy environment. Yue and Zhang (2020) and Liang et al. (2018) considered the hesitancy of the subjects and developed TSMDM approaches, where the evaluations are hesitant fuzzy sets. Although the evaluations of preference ordinal are qualitative, the expression of which is not simple enough for subjects. Except the preference ordinal, the above mentioned evaluations are all quantitative, which is complicated for subjects to express. Zadeh 
(1975) pointed out the linguistic information can be described as the linguistic variables, which are quantitative mathematical symbols. On the basis of this, $\mathrm{Xu}$ (2012) introduces the linguistic terms (LTs). The LTs are simple enough for subjects to express their qualitative evaluations. In other words, the qualitative information is quantitatively expressed by using the LTs. To take into account the linguistic information of subjects, Lin et al. (2018) introduced an enhanced TSMDM approach, where the evaluations are 2-tuple linguistic preferences.

Rodriguez et al. (2012) defined hesitant fuzzy linguistic term sets (HFLTSs) based on hesitant fuzzy sets and the LTs. The HFLTSs can effectively express the qualitative information in a hesitant condition, where the subjects prefer to give several LTs. Pang et al. (2016) pointed out the drawback of the HFLTSs that the probabilities of the involved LTs are identical, they further defined probabilistic linguistic term sets (PLTSs), in which the probabilities can be varied by subjects according to their preference and degree of belief. PLTSs are mainly implemented to describe the evaluations in decision-making problems. Wang et al. (2019) introduced two distances of the PLTSs and further proposed a distance-based multi-criteria group decision-making approach. Liu et al. (2018) presented several entropy measures for the PLTSs, including the fuzzy entropy, the hesitant entropy and the total entropy. Liao et al. (2017) established a linear programming method for multicriteria decision-making, where the evaluations are PLTSs. Gou and Xu (2016) defined several novel basic operational laws for the PLTSs based on equivalent transformation function. By considering the disparity degrees of the LTs, Liao et al. (2019) also defined several novel operational laws for the PLTSs. Wu et al. (2018) proposed a multi-criteria decision-making method based on the expectation function of the PLTSs and the improved Borda rule. Kobina et al. (2017) defined the probabilistic linguistic power aggregation operators, on the basis of this, they further extracted a multi-criteria group decision-making method. The application of the PLTSs in the decision-making methods is proved to be effective. Since the subjects are mainly human who prefer to conveniently give their oral evaluations, it is necessary to introduce the PLTSs to the TSMDM approaches.

Comparison is usually used for decision-making. Among existing decision-making approaches, the preference ranking organization method (PROMETHEE) is a typical one for comparison. Brans and Vinckes (1985) introduced the PROMETHEE to ensure it is easily accepted and understood by the decision-maker. Later, several modified decision-making methods were developed to extend the PROMETHEE (Chen et al. 2011; Dağdeviren 2008; Vinodh and Girubha 2012; Vujosevic and 
Popovic 2016). The objects are compared in PROMETHEE under six different criteria, which is the most outstanding feature. However, the above mentioned PROMETHEE are developed under the real number environment and did not consider the fuzzy condition. It is worthy to note that Liu and Li (2018) and Yu et al. (2019) introduced the PROMETHEE, where the evaluations are the PLTSs. The case studies in these two decision-making methods combining the PROMETHEE and the PLTSs exhibit that the application of the PLTSs is more precise and convenient when comparing several objects.

On the basis of these analyses, we propose a TSMDM approach based on PROMETHEE, where the evaluations are the PLTSs. The contributions of this paper are summarized as follows:

(1) The preference functions for the PLTSs are defined based on the PROMETHEE.

(2) A novel matching aspiration model is built.

(3) A TSMDM approach under the probabilistic linguistic environment is proposed.

The rest of this paper is organized as follows: Section 2 reviews several basic concepts of the PLTSs and the two-sided matching. Section 3 proposes a TSMDM approach based on PROMETHEE in details. Section 4 exhibits an illustrative example by using the proposed approach. Section 5 conducts sensitivity analysis and comparative analysis. Conclusion is pointed out in Section 6.

\section{Preliminaries}

In this section, several basic concepts with respect to the PLTSs and the two-sided matching are reviewed.

\subsection{PLTSs}

$\mathrm{Xu}$ (2012) defined the linguistic term set (LTS) as

$$
S=\left\{s_{\varsigma} \mid \varsigma=-\tau, \mathrm{L},-1,0,1, \mathrm{~L}, \tau\right\}
$$

where the mid linguistic term $s_{0}$ represents an assessment of "indifference", $\tau$ is an integer and $\tau \geq 1$, and with the rest of the linguistic terms being placed symmetrically around it. LTs $s_{\varsigma_{1}}, s_{\varsigma_{2}} \in S$ and satisfy two conditions: 
(1) If $\varsigma_{1}>\varsigma_{2}$, then $s_{\varsigma_{1}}>s_{\varsigma_{2}}$;

(2) The negation operator is defined: $n e g\left(s_{S_{1}}\right)=s_{-s_{1}}$, especially, $n e g\left(s_{0}\right)=s_{0}$.

The operational laws of the LTs $s_{\varsigma_{1}}, s_{S_{2}} \in S$ are also defined by Xu (2012) as:

(1) $s_{S_{1}} \oplus s_{S_{2}}=s_{S_{1}+\varsigma_{2}}$;

(2) $\lambda s_{\varsigma_{1}}=s_{\lambda \varsigma_{1}}$.

By considering the hesitancy of the evaluator and the distribution of probabilities, Pang et al. (2016) defined the PLTSs.

Definition 1 (Pang et al. 2016). Let $S=\left\{s_{\varsigma} \mid \varsigma=-\tau, \mathrm{L},-1,0,1, \mathrm{~L}, \tau\right\}$ be a LTS, a PLTS can be defined as:

$$
L(p)=\left\{L^{(l)}\left(p^{(l)}\right) \mid L^{(l)} \in S, p^{(l)} \geq 0, l=1,2, \mathrm{~L}, \# L(p), \sum_{l=1}^{\# L(p)} p^{(l)} \leq 1\right\},
$$

where $L^{(l)}\left(p^{(l)}\right)$ is the LT $L^{(l)}$ associated with the probability $p^{(l)}$, and $\# L(p)$ is the number of all different linguistic terms in $L(p)$.

Definition 2 (Pang et al. 2016). Given a PLTS $L(p)=\left\{L^{(l)}\left(p^{(l)}\right) \mid l=1,2, \mathrm{~L}, \# L(p)\right\}$, and $\varsigma^{(l)}$ is the subscript of LT $L^{(l)} . L(p)$ is called an ordered PLTS, if the elements $L^{(l)}\left(p^{(l)}\right)(l=1,2, \mathrm{~L}, \# L(p)) \quad$ are arranged according to the values of $\varsigma^{(l)} p^{(l)}(l=1,2, \mathrm{~L}, \# L(p))$ in descending order.

For the PLTS $L(p)=\left\{L^{(l)}\left(p^{(l)}\right) \mid l=1,2, \mathrm{~L}, \# L(p)\right\}$ where $\sum_{l=1}^{\# L(p)} p^{(l)}<1$, Pang et al. (2016) assigned the ignored probability to the LTs in $L(p)$ averagely.

Definition 3 (Pang et al. 2016). Given a PLTS $L(p)$ with $\sum_{l=1}^{\# L(p)} p^{(l)}<1$, then the associated PLTS $L(\bar{p})$ is defined by

$$
L(\bar{p})=\left\{L^{(l)}\left(\bar{p}^{(l)}\right) \mid l=1,2, \mathrm{~L}, \# L(\bar{p})\right\},
$$


where $\bar{p}^{(l)}=\frac{p^{(l)}}{\# L(p)}$, for all $l=1,2, \mathrm{~L}, \# L(\bar{p})$.

$$
\sum_{l=1}^{\# L(p)} p^{(l)}
$$

The trouble may occure when operating two PLTSs where the numbers of the LTs are not identical. Pang e al. (2016) pointed out that for two PLTSs $L_{1}\left(p_{1}\right)$ and $L_{2}\left(p_{2}\right)$ where the number of the LTs in the former is $\# L_{1}\left(p_{1}\right)$ and that in the later is $\# L_{2}\left(p_{2}\right)$, if $\# L_{1}\left(p_{1}\right)>\# L_{2}\left(p_{2}\right)$, then $\left(\# L_{1}\left(p_{1}\right)-\# L_{2}\left(p_{2}\right)\right)$ smallest LTs of $L_{2}\left(p_{2}\right)$ associated with zero probabilities should be added to $L_{2}\left(p_{2}\right)$. We call a PLTS that has been ordered, assigned the ignored probability, added the smallest LT the normalized PLTSs.

Definition 4 (Pang et al. 2016). Let $L(p)=\left\{L^{(l)}\left(p^{(l)}\right) \mid l=1,2, \mathrm{~L}, \# L(p)\right\}$ be a PLTS and $\varsigma^{(l)}$ be the subscript of $L^{(l)}$. Then the score of $L(p)$ is $E(L(p))=s_{-}$, where $\bar{\varsigma}=\frac{\sum_{l=1}^{\# L(p)} \varsigma^{(l)} p^{(l)}}{\sum_{l=1}^{\# L(p)} p^{(l)}}$. The deviation degree of $L(p)$ is $\sigma(L(p))=\frac{\left(\sum_{l=1}^{\# L(p)}\left(p^{(l)}\left(\varsigma^{(l)}-\bar{\varsigma}\right)\right)^{2}\right)^{\frac{1}{2}}}{\sum_{l=1}^{\# L(p)} p^{(l)}}$.

Based on the mean-variance rule, Pang et al. (2016) defined the comparison method for the PLTSs.

Definition 5 (Pang et al. 2016). For two PLTSs $L_{1}\left(p_{1}\right)$ and $L_{2}\left(p_{2}\right)$,

(1) if $E\left(L_{1}\left(p_{1}\right)\right)>E\left(L_{2}\left(p_{2}\right)\right)$, then $L_{1}\left(p_{1}\right)>L_{2}\left(p_{2}\right)$;

(2) if $E\left(L_{1}\left(p_{1}\right)\right)=E\left(L_{2}\left(p_{2}\right)\right)$, then

(a) if $\sigma\left(L_{1}\left(p_{1}\right)\right)<\sigma\left(L_{2}\left(p_{2}\right)\right)$, then $L_{1}\left(p_{1}\right)>L_{2}\left(p_{2}\right)$;

(b) if $\sigma\left(L_{1}\left(p_{1}\right)\right)=\sigma\left(L_{2}\left(p_{2}\right)\right)$, then $L_{1}\left(p_{1}\right)=L_{2}\left(p_{2}\right)$.

The comparison method proposed by Pang et al. (2016) can only reflect the qualitative relationship between two PLTSs, the quantitative difference between them cannot be obtained. Lin and $\mathrm{Xu}$ (2017) defined the normalized Hamming distance for the PLTSs.

Definition 6 (Lin and $\mathrm{Xu}$ 2017). Let $S=\left\{s_{\varsigma} \mid \varsigma=-\tau, \mathrm{L},-1,0,1, \mathrm{~L}, \tau\right\}$, 
$L_{1}\left(p_{1}\right)=\left\{L_{1}^{(l)}\left(p_{1}^{(l)}\right) \mid l=1,2, \mathrm{~L}, \# L_{1}\left(p_{1}\right)\right\}$

and

$L_{2}\left(p_{2}\right)=\left\{L_{2}{ }^{(l)}\left(p_{2}{ }^{(l)}\right) \mid l=1,2, \mathrm{~L}, \# L_{2}\left(p_{2}\right)\right\} \quad$ be $\quad$ two $\quad$ PLTSs $\quad$ on $\quad S \quad$ and $\# L_{1}\left(p_{1}\right)=\# L_{2}\left(p_{2}\right)$, then the normalized Hamming distance between them is defined as:

$$
d\left(L_{1}\left(p_{1}\right), L_{2}\left(p_{2}\right)\right)=\frac{1}{\# L_{1}\left(p_{1}\right)} \sum_{l=1}^{\# L_{1}\left(p_{1}\right)}\left|p_{1}^{(l)} \times \frac{\varsigma_{1}^{(l)}}{2 \tau}-p_{2}^{(l)} \times \frac{\varsigma_{2}^{(l)}}{2 \tau}\right|,
$$

where $\varsigma_{1}^{(l)}$ and $\varsigma_{2}^{(l)}$ are the subscripts of the LTs $L_{1}^{(l)}$ and $L_{2}^{(l)}$ respectively.

\subsection{Two-sided Matching}

Let $A=\left\{A_{i} \mid i \in M\right\}(M=\{1,2, \mathrm{~L}, m\})$ and $B=\left\{B_{j} \mid j \in N\right\}(N=\{1,2, \mathrm{~L}, n\})$ be two subject sets. Without loss of generality, we let $n \geq m$.

Definition 7 (Lin et al. 2018). A matching is a mapping $\Upsilon: A \mathrm{U} B \rightarrow A \mathrm{U} B \mathrm{U}\{\phi\}$ for all $\forall A_{i} \in A$ and $\forall B_{j} \in B$ such that

(1) $\Upsilon\left(A_{i}\right) \in B U\{\phi\}$,

(2) $\Upsilon\left(B_{j}\right) \in A U\{\phi\}$,

(3) $\Upsilon\left(A_{i}\right)=B_{j}$, if and only if $\Upsilon\left(B_{j}\right) \in A_{i}$.

If $\Upsilon\left(A_{i}\right)=B_{j}$, we call $\left(A_{i}, B_{j}\right)$ a matching pair. All matching pairs consist of a matching scheme. Condition (1) represents $A_{i}$ matches a subject from side $B$ or does not match a subject. Condition (2) represents $B_{j}$ matches a subject from side $A$ or does not match a subject. Condition (3) represents $A_{i}$ matches $B_{j}$ if and only if $B_{j}$ matches $A_{i}$, which also means the mapping is a one to one matching that if $A_{i}$ matches $B_{j}$, then $B_{j}$ cannot be matched by the subjects from the side $A$. The mapping $\Upsilon$ is conducted by the intermediary, which is not usually the third part. In some conditions such as the matching of personnel and jobs, the company not only conducts the matching process, but also participates into the matching as the side of jobs. The intermediary is considered to be the third part in this research. The main task of the TSMDM 
approaches is to match two sides of subjects one by one and make the matching scheme satisfy all subjects.

Remark 1. The conditions (1) and (2) in the Definition 7 imply that the subjects may not be totally matched, i.e., the number of the matching pairs is less than $m$. In such cases, there remain some subjects from side $A$ and $B$ that are not matched, which violate the task of the TSMDM approaches. Since the number of the subjects from side $A$ is less than that of side $B$, i.e. $n \geq m$, we redefine the condition (1) in Definition 7 as $\Upsilon\left(A_{i}\right) \in B$ to ensure each subjects from side $A$ can be matched.

\section{TSMDM Approach}

The TSMDM problem is firstly described in this section, then we propose a TSMDM approach.

\subsection{Description of TSMDM Problem}

The subject sets $A$ and $B$ are identical to the subject sets in Definition 7. The criteria set $C^{1}=\left\{C_{\mu}^{1} \mid \mu \in R\right\}(R=\{1,2, \mathrm{~L}, r\}, r \geq 2) \quad$ is related to side $A$, while criteria set $C^{2}=\left\{C_{v}^{2} \mid v \in T\right\}(T=\{1,2, \mathrm{~L}, t\}, t \geq 2)$ is related to side $B$. The weight vectors related to $C^{1}$ and $C^{2}$ are $\omega^{1}=\left(\omega_{1}^{1}, \omega_{2}^{1}, \mathrm{~L}, \omega_{r}^{1}\right)^{\mathrm{T}}$ and $\omega^{2}=\left(\omega_{1}^{2}, \omega_{2}^{2}, \mathrm{~L}, \omega_{t}^{2}\right)^{\mathrm{T}}$ respectively. The evaluation of $B_{j}$ to $A_{i}$ under the criterion $C_{\mu}^{1}$ and the evaluation of $A_{i}$ to $B_{j}$ under the criterion $C_{v}^{2}$ are denoted as $e_{\mu, i j}^{1}$ and $e_{v, i j}^{2}$, respectively. All $e_{\mu, i j}^{1}$ construct the evaluation matrix $E^{1}=\left[e_{\mu, i j}^{1}\right]_{(m \times r) \times n}$, while $e_{v, i j}^{2}$ construct the evaluation matrix $E^{2}=\left[e_{v, i j}^{2}\right]_{m \times(n \times t)}$. The evaluations are all PLTSs. To solve this problem is to find an optimal matching scheme for sides $A$ and $B$ by considering the above information.

\subsection{Matching Process}

The criteria in decision-making problems can be sorted into two kinds: benefit types and cost types. A higher evaluation is better for the benefit criterion, while a lower one is better for the cost criterion. For a group of criteria including benefit ones and cost ones, the identical operations for 
the evaluations under these criteria can cause confusion. Hence, transforming the cost type of evaluations to the benefit type is necessary. For the evaluation $L(p)=\left\{L^{(l)}\left(p^{(l)}\right) \mid l=1,2, \mathrm{~L}, \# L(p)\right\}$, if it is under the benefit criteria, then holds its value, if it is under the cost criteria, then the involved LTs should be transformed by means of their negation operator. We further define a transforming function as:

$$
g(L(p))=\left\{\begin{array}{l}
\left\{L^{(l)}\left(p^{(l)}\right) \mid l=1,2, \mathrm{~L}, \# L(p)\right\}, \text { if } L(p) \text { is in benefit type, } \\
\left\{n e g\left(L^{(l)}\right)\left(p^{(l)}\right) \mid l=1,2, \mathrm{~L}, \# L(p)\right\}, \text { if } L(p) \text { is in cost type. }
\end{array}\right.
$$

Brans and Vincke (1985) classified the criteria into six types and defined distinct preference function for them. However, the condition they considered is that the evaluations are real numbers and their difference can be easily measured by the subtraction operator. The operation is not that simple in the fuzzy environment. The subtraction operator is not defined for the PLTSs. The contribution of the subtraction operator is to calculate the difference between two real numbers. Distances are usually used for measuring the difference between two PLTSs. We extend the preference functions to accommodate the probabilistic linguistic environment based on distance. On the aspect of evaluations of $B_{j}$ to $A_{i_{1}}$ and $B_{j}$ to $A_{i_{2}}, j \in N, i_{1}, i_{2} \in M$, the distance-based preference functions are defined in six conditions.

(1) If $C_{\mu}^{1}$ is a Usual Criterion, then

$$
f^{11}\left(e_{\mu, i_{1} j}^{1}, e_{\mu, i_{2} j}^{1}\right)=\left\{\begin{array}{l}
0, \text { if } e_{\mu, i_{1} j}^{1} \leq e_{\mu, i_{2} j}^{1} \\
1, \text { if } e_{\mu, i_{1} j}^{1}>e_{\mu, i_{2} j}^{1}
\end{array}\right.
$$

(2) If $C_{\mu}^{1}$ is a Quasi-Criterion, then

$$
f^{12}\left(e_{\mu, i_{1} j}^{1}, e_{\mu, i_{2} j}^{1}\right)=\left\{\begin{array}{l}
0, \text { if } e_{\mu, i_{1} j}^{1} \leq e_{\mu, i_{2} j}^{1}, \\
0, \text { if } e_{\mu, i_{1} j}^{1}>e_{\mu, i_{2} j}^{1} \text { and } d\left(e_{\mu, i_{1} j}^{1}, e_{\mu, i_{2} j}^{1}\right) \leq \alpha^{1}, \\
1, \text { if } e_{\mu, i_{1} j}^{1}>e_{\mu, i_{2} j}^{1} \text { and } d\left(e_{\mu, i_{1} j}^{1}, e_{\mu, i_{2} j}^{1}\right)>\alpha^{1} .
\end{array}\right.
$$

(3) If $C_{\mu}^{1}$ is a Criterion with Linear Preference, then 


$$
f^{13}\left(e_{\mu, i_{1} j}^{1}, e_{\mu, i_{2} j}^{1}\right)=\left\{\begin{array}{l}
0, \text { if } e_{\mu, i_{1} j}^{1} \leq e_{\mu, i_{2} j}^{1}, \\
\frac{d\left(e_{\mu, i_{1} j}^{1}, e_{\mu, i_{2} j}^{1}\right)}{\beta}, \text { if } e_{\mu, i_{1} j}^{1}>e_{\mu, i_{2} j}^{1} \text { and } d\left(e_{\mu, i_{1} j}^{1}, e_{\mu, i_{2} j}^{1}\right)<\beta^{1} \\
1, \text { if } e_{\mu, i_{1} j}^{1}>e_{\mu, i_{2} j}^{1} \text { and } d\left(e_{\mu, i_{1} j}^{1}, e_{\mu, i_{2} j}^{1}\right) \geq \beta^{1}
\end{array}\right.
$$

(4) If $C_{\mu}^{1}$ is a Level-Criterion, then

$$
f^{14}\left(e_{\mu, i_{1} j}^{1}, e_{\mu, i_{2} j}^{1}\right)=\left\{\begin{array}{l}
0, \text { if } e_{\mu, i_{1} j}^{1} \leq e_{\mu, i_{2} j}^{1}, \\
0, \text { if } e_{\mu, i_{1} j}^{1}>e_{\mu, i_{2} j}^{1} \text { and } d\left(e_{\mu, i_{1} j}^{1}, e_{\mu, i_{2} j}^{1}\right)<\delta^{1}, \\
\frac{1}{2}, \text { if } e_{\mu, i_{1} j}^{1}>e_{\mu, i_{2} j}^{1} \text { and } \delta^{1}<d\left(e_{\mu, i_{1} j}^{1}, e_{\mu, i_{2} j}^{1}\right)<\delta^{1}+\phi^{1}, \\
1, \text { if } e_{\mu, i_{1} j}^{1}>e_{\mu, i_{2} j}^{1} \text { and } d\left(e_{\mu, i_{1} j}^{1}, e_{\mu, i_{2} j}^{1}\right)>\delta^{1}+\phi^{1} .
\end{array}\right.
$$

(5) If $C_{\mu}^{1}$ is a Criterion with Linear Preference and Indifference, then

$$
f^{15}\left(e_{\mu, i_{1} j}^{1}, e_{\mu, i_{2} j}^{1}\right)=\left\{\begin{array}{l}
0, \text { if } e_{\mu, i_{1} j}^{1} \leq e_{\mu, i_{2} j}^{1}, \\
0, \text { if } e_{\mu, i_{1} j}^{1}>e_{\mu, i_{2} j}^{1} \text { and } d\left(e_{\mu, i_{1} j}^{1}, e_{\mu, i_{2} j}^{1}\right) \leq \varphi^{1}, \\
\frac{d\left(e_{\mu, i_{j} j}^{1}, e_{\mu, i_{2} j}^{1}\right)-\varphi^{1}}{\eta^{1}}, \text { if } e_{\mu, i_{1} j}^{1}>e_{\mu, i_{2} j}^{1} \text { and } \varphi^{1}<d\left(e_{\mu, i_{1} j}^{1}, e_{\mu, i_{2} j}^{1}\right)<\varphi^{1}+\eta^{1}, \\
1, \text { if } e_{\mu, i_{1} j}^{1}>e_{\mu, i_{2} j}^{1} \text { and } d\left(e_{\mu, i_{1} j}^{1}, e_{\mu, i_{2} j}^{1}\right) \geq \varphi^{1}+\eta^{1} .
\end{array}\right.
$$

(6) If $C_{\mu}^{1}$ is a Gaussian Criterion, then

$$
f^{16}\left(e_{\mu, i_{1} j}^{1}, e_{\mu, i_{2} j}^{1}\right)=\left\{\begin{array}{l}
0, \text { if } e_{\mu, i_{1} j}^{1} \leq e_{\mu, i_{2} j}^{1}, \\
1-e^{-\frac{\left(d\left(e_{\mu, i_{j} j}^{1}, e_{\mu, i_{2} j}^{1}\right)\right)^{2}}{2 \pi}}, \text { if } e_{\mu, i_{1} j}^{1}>e_{\mu, i_{2} j}^{1} .
\end{array}\right.
$$

For the distance-based function $f^{1}(\cdot) \in\left\{f^{11}(\cdot), f^{12}(\cdot), f^{13}(\cdot), f^{14}(\cdot), f^{15}(\cdot), f^{16}(\cdot)\right\}, \quad \alpha^{1}>0, \quad \beta^{1}>0, \delta^{1}>0, \phi^{1} \geq 0$, $\varphi^{1}>0, \eta^{1}>0$, the value of $\pi$ is the distance between the origin and the point of inflexion of the curve. $f^{1}\left(e_{\mu, i_{1} j}^{1}, e_{\mu, i_{2} j}^{1}\right)$ denotes the preference degree of $B_{j}$ to $A_{i_{1}}$ compared to $A_{i_{2}}$ under the criterion $C_{\mu}^{1}$. Since the criteria are all considered to be the benefit types, for six distancebased preference functions, $f^{1}\left(e_{\mu, i_{1} j}^{1}, e_{\mu, i_{2} j}^{1}\right)=0$ if $e_{\mu, i_{1} j}^{1}<e_{\mu, i_{2} j}^{1}$. If $e_{\mu, i_{1} j}^{1}>e_{\mu, i_{2} j}^{1}$, and the distance between them is large enough to be larger than a non-negative number, 
$f^{1}\left(e_{\mu, i_{1} j}^{1}, e_{\mu, i_{2} j}^{1}\right)=1$. Especially, this non-negative number for usual criteria is equal to zero, while it should be larger than a sufficiently large number for Gaussian criteria. For other conditions, the value of $f^{1}\left(e_{\mu, i_{1} j}^{1}, e_{\mu, i_{2} j}^{1}\right)$ is flexibly varied according to the type of criteria. Owing to the evaluations are represented by the PLTSs rather than the real numbers, the distance of PLTSs is utilized to replace the subtraction of real numbers. Take the condition $e_{\mu, i_{1} j}^{1}-e_{\mu, i_{2} j}^{1}>\alpha^{1}$ as an example, it can be easily expressed by the subtraction of real numbers, however, cannot be straightforwardly expressed by the PLTSs. Since $\alpha^{1}>0$, we divide this condition into two conditions, $e_{\mu, i_{1} j}^{1}-e_{\mu, i_{2} j}^{1}>0$ and the difference between them is larger than $\alpha^{1}$. $e_{\mu, i_{1} j}^{1}-e_{\mu, i_{2} j}^{1}>0$ is transformed to $e_{\mu, i_{1} j}^{1}>e_{\mu, i_{2} j}^{1}$, which means $e_{\mu, i_{1} j}^{1}$ is superior to $e_{\mu, i_{2} j}^{1}$. This relation can be obtained by using the method introduced in Definition 5. The difference is measured by the distance introduced in Definition 6. Hence, the six preference function are extended to the probabilistic linguistic environment. Since the evaluations are all transformed to the benefit types, there is no need to define the preference functions for cost criteria.

Simultaneously, on the aspect of evaluation of $A_{i}$ to $B_{j_{1}}$ and $A_{i}$ to $B_{j_{2}}$, the distancebased preference functions can also be defined.

(1) If $C_{v}^{2}$ is a Usual Criterion, then

$$
f^{21}\left(e_{v, i j_{1}}^{2}, e_{v, i j_{2}}^{2}\right)=\left\{\begin{array}{l}
0, \text { if } e_{v, i j_{1}}^{2} \leq e_{v, i j_{2}}^{2}, \\
1, \text { if } e_{v, i j_{1}}^{2}>e_{v, i j_{2}}^{2} .
\end{array}\right.
$$

(2) If $C_{v}^{2}$ is a Quasi-Criterion, then

$$
f^{22}\left(e_{v, i j_{1}}^{2}, e_{v, i j_{2}}^{2}\right)=\left\{\begin{array}{l}
0, \text { if } e_{v, i j_{1}}^{2} \leq e_{v, i j_{2}}^{2}, \\
0, \text { if } e_{v, i j_{1}}^{2}>e_{v, i j_{2}}^{2} \text { and } d\left(e_{v, i j_{1}}^{2}, e_{v, i j_{2}}^{2}\right) \leq \alpha^{2}, \\
1, \text { if } e_{v, i j_{1}}^{2}>e_{v, i j_{2}}^{2} \text { and } d\left(e_{v, i j_{1}}^{2}, e_{v, i j_{2}}^{2}\right)>\alpha^{2} .
\end{array}\right.
$$

(3) If $C_{v}^{2}$ is a Criterion with Linear Preference, then 


$$
f^{23}\left(e_{v, i j_{1}}^{2}, e_{v, i j_{2}}^{2}\right)=\left\{\begin{array}{l}
0, \text { if } e_{v, i j_{1}}^{2} \leq e_{v, i j_{2}}^{2}, \\
\frac{d\left(e_{v, i j_{1}}^{2}, e_{v, i j_{2}}^{2}\right)}{\beta}, \text { if } e_{v, i j_{1}}^{2}>e_{v, i j_{2}}^{2} \text { and } d\left(e_{v, i j_{1}}^{2}, e_{v, i j_{2}}^{2}\right)<\beta^{2}, \\
1, \text { if } e_{v, i j_{1}}^{2}>e_{v, i j_{2}}^{2} \text { and } d\left(e_{v, i j_{1}}^{2}, e_{v, i j_{2}}^{2}\right) \geq \beta^{2} .
\end{array}\right.
$$

(4) If $C_{v}^{2}$ is a Level-Criterion, then

$$
f^{24}\left(e_{v, i j_{1}}^{2}, e_{v, i j_{2}}^{2}\right)=\left\{\begin{array}{l}
0, \text { if } e_{v, i j_{1}}^{2} \leq e_{v, i j_{2}}^{2}, \\
0, \text { if } e_{v, i j_{1}}^{2}>e_{v, i j_{2}}^{2} \text { and } d\left(e_{v, i j_{1}}^{2}, e_{v, i j_{2}}^{2}\right)<\delta^{2}, \\
\frac{1}{2}, \text { if } e_{v, i j_{1}}^{2}>e_{v, i j_{2}}^{2} \text { and } \delta^{2}<d\left(e_{v, i j_{1}}^{2}, e_{v, i j_{2}}^{2}\right)<\delta^{2}+\phi^{2}, \\
1, \text { if } e_{v, i j_{1}}^{2}>e_{v, i j_{2}}^{2} \text { and } d\left(e_{v, i j_{1}}^{2}, e_{v, i j_{2}}^{2}\right)>\delta^{2}+\phi^{2} .
\end{array}\right.
$$

(5) If $C_{v}^{2}$ is a Criterion with Linear Preference and Indifference, then

$$
f^{25}\left(e_{v, i j_{1}}^{2}, e_{v, i j_{2}}^{2}\right)=\left\{\begin{array}{l}
0, \text { if } e_{v, i j_{1}}^{2} \leq e_{v, i j_{2}}^{2}, \\
0, \text { if } e_{v, i j_{1}}^{2}>e_{v, i j_{2}}^{2} \text { and } d\left(e_{v, i j_{1}}^{2}, e_{v, i j_{2}}^{2}\right) \leq \varphi^{2}, \\
\frac{d\left(e_{v, i j_{1}}^{2}, e_{v, i j_{2}}^{2}\right)-\varphi^{1}}{\eta^{1}}, \text { if } e_{v, i j_{1}}^{2}>e_{v, i j_{2}}^{2} \text { and } \varphi^{2}<d\left(e_{v, i j_{1}}^{2}, e_{v, i j_{2}}^{2}\right)<\varphi^{2}+\eta^{2}, \\
1, \text { if } e_{v, i j_{1}}^{2}>e_{v, i j_{2}}^{2} \text { and } d\left(e_{v, i j_{1}}^{2}, e_{v, i j_{2}}^{2}\right) \geq \varphi^{2}+\eta^{2} .
\end{array}\right.
$$

(6) If $C_{v}^{2}$ is a Gaussian Criterion, then

$$
f^{26}\left(e_{v, i_{1}}^{2}, e_{v, i j_{2}}^{2}\right)=\left\{\begin{array}{l}
0, \text { if } e_{v, i j_{1}}^{2} \leq e_{v, i j_{2}}^{2}, \\
1-e^{-\frac{\left(d\left(e_{v, i j_{1}}^{2}, e_{v, j_{2}}^{2}\right)\right)^{2}}{2 \pi}}, \text { if } e_{v, i j_{1}}^{2}>e_{v, i j_{2}}^{2} .
\end{array}\right.
$$

For the distance-based function $f^{2}(\cdot) \in\left\{f^{21}(\cdot), f^{22}(\cdot), f^{23}(\cdot), f^{24}(\cdot), f^{25}(\cdot), f^{26}(\cdot)\right\}, \quad \alpha^{2}>0, \quad \beta^{2}>0, \quad \delta^{2}>0$, $\phi^{2} \geq 0, \varphi^{2}>0, \eta^{2}>0$, the value of $\pi$ is the distance between the origin and the point of inflexion of the curve. $f^{2}\left(e_{v, i j_{1}}^{2}, e_{v, i j_{2}}^{2}\right)$ denotes the preference degree of $A_{i}$ to $B_{j_{1}}$ compared to $B_{j_{2}}$ under the criterion $C_{v}^{2}$.

$f^{1}\left(e_{\mu, i_{1} j}^{1}, e_{\mu, i_{2} j}^{1}\right)$ and $f^{2}\left(e_{v, i_{1}}^{2}, e_{v, i_{2}}^{2}\right)$ can be integrated into the preference index $h^{1}$ and $h^{2}$ respectively. 


$$
\begin{aligned}
& h_{j}^{1}\left(A_{i_{1}}, A_{i_{2}}\right)=\sum_{\mu=1}^{r} \omega_{\mu}^{1} f^{1}\left(e_{\mu, i_{1} j}^{1}, e_{\mu, i_{2} j}^{1}\right), \\
& h_{i}^{2}\left(B_{j_{1}}, B_{j_{2}}\right)=\sum_{v=1}^{t} \omega_{v}^{2} f^{2}\left(e_{v, i_{1}}^{2}, e_{v, i_{2}}^{2}\right),
\end{aligned}
$$

where $h_{j}^{1}\left(A_{i_{1}}, A_{i_{2}}\right)$ is the preference index of $B_{j}$ to $A_{i_{1}}$ compared to $A_{i_{2}}, h_{i}^{2}\left(B_{j_{1}}, B_{j_{2}}\right)$ is the preference index of $A_{i}$ to $B_{j_{1}}$ compared to $B_{j_{2}}, \quad \omega^{1}=\left(\omega_{1}^{1}, \omega_{2}^{1}, \mathrm{~L}, \omega_{r}^{1}\right)^{\mathrm{T}}$ and $\omega^{2}=\left(\omega_{1}^{2}, \omega_{2}^{2}, \mathrm{~L}, \omega_{t}^{2}\right)^{\mathrm{T}}$ are the weight vectors related to $C^{1}$ and $C^{2}$ respectively. Note that the values of $h_{j}^{1}\left(A_{i_{1}}, A_{i_{2}}\right)$ and $h_{i}^{2}\left(B_{j_{1}}, B_{j_{2}}\right)$ are all larger or equal to zero.

The outgoing flows can be defined as:

$$
\begin{aligned}
& a_{i_{1} j}^{+}=\sum_{i_{1} \neq i_{2}} h_{j}^{1}\left(A_{i_{1}}, A_{i_{2}}\right), \\
& b_{i j_{1}}^{+}=\sum_{j_{1} \neq j_{2}} h_{i}^{2}\left(B_{j_{1}}, B_{j_{2}}\right) .
\end{aligned}
$$

Similarly, the incoming flows can be defined as:

$$
\begin{aligned}
& a_{i_{1} j}^{-}=\sum_{i_{1} \neq i_{2}} h_{j}^{1}\left(A_{i_{2}}, A_{i_{1}}\right), \\
& b_{i j_{1}}^{-}=\sum_{j_{1} \neq j_{2}} h_{i}^{2}\left(B_{j_{2}}, B_{j_{1}}\right) .
\end{aligned}
$$

Subsequently, the net-flows are defined as:

$$
\begin{aligned}
& a_{i_{1} j}=a_{i_{1} j}^{+}-a_{i_{1} j}^{-}, \\
& b_{i j_{1}}=b_{i j_{1}}^{+}-b_{i j_{1}}^{-} .
\end{aligned}
$$

Since the higher the $a_{i_{1} j}$ is, the higher the satisfaction of $B_{j}$ to $A_{i_{1}}$ will be, we redefine $a_{i_{1} j}$ and $b_{i j_{1}}$ as the satisfaction degrees.

Yue et al. (2016) pointed out the matching aspiration is important to measure the success rate for matching two subjects from two sides. The larger $\frac{1}{\left|a_{i j}-b_{i j}\right|}$ is, the greater the success rate for matching $A_{i}$ with $B_{j}$ will be. In this case, the matching aspiration between $A_{i}$ and $B_{j}$ should be large. On the contrast, a lower value of $\frac{1}{\left|a_{i j}-b_{i j}\right|}$ indicates the difference between $a_{i j}$ 
and $b_{i j}$ is large, which also indicates the success rate for matching $A_{i}$ with $B_{j}$ is lower. We further illustrate the matching aspiration by an extreme case. Let the interval $[\bar{a}, \underline{a}]$ be the scale of the satisfaction degrees, where $\bar{a}$ is the upper bound and $\underline{a}$ is the lower bound, the satisfaction degrees of two sides $a_{i j}$ and $b_{i j}$ satisfy $\bar{a} \leq a_{i j} \leq \underline{a}$ and $\bar{a} \leq b_{i j} \leq \underline{a}$ respectively. It is obvious that the largest difference between $a_{i j}$ and $b_{i j}$ is $\bar{a}-\underline{a}$, which means for a pair of subjects $A_{i}$ and $B_{j}$, a subject is totally satisfied by the other while the other is totally dissatisfied by this subject. Subsequently, the success rate for matching these two subjects are the lowest owing to the totally dissatisfaction. By introducing the matching aspiration $\theta_{i j}$ and 0,1 variable $x_{i j}$, the multi-objectives TSMDM model $(M 1)$ can be built as:

$$
(M 1)\left\{\begin{array}{r}
\max Z^{A}=\sum_{i=1}^{m} \sum_{j=1}^{n} \theta_{i j} a_{i j} x_{i j}, \\
\max Z^{B}=\sum_{j=1}^{n} \sum_{i=1}^{m} \theta_{i j} b_{i j} x_{i j}, \\
\text { s.t. } \quad \sum_{j=1}^{n} x_{i j}=1, i=1,2, \mathrm{~L}, m, \\
\sum_{i=1}^{m} x_{i j} \leq 1, j=1,2, \mathrm{~L}, n,
\end{array}\right.
$$

where $x_{i j}=0$ denotes $A_{i}$ does not matches $B_{j}, x_{i j}=1$ denotes $A_{i}$ matches $B_{j}$, the goal functions in equations (23) and (24) aim to maximize the satisfaction degrees of sides $A$ and $B$ respectively, equation (25) depicts $A_{i}$ should be matched, equation (26) depicts $B_{j}$ may not be matched.

To calculate the matching aspiration $\theta_{i j}$, we build $(M 2)$ as:

$$
(M 2)\left\{\begin{array}{c}
\max Z^{\prime}=\sum_{i=1}^{m} \sum_{j=1}^{n} \frac{\theta_{i j}}{e^{\left|a_{i j}-b_{i j \mid}\right|}}, \\
\text { s.t. } \quad \sum_{i=1}^{m} \sum_{j=1}^{n}\left(\theta_{i j}\right)^{2}=1,0 \leq \theta_{i j} \leq 1,
\end{array}\right.
$$

where the goal function in equation (27) represents the comprehensive reciprocal difference of two sides. Equation (28) is the restriction for matching aspiration. To maximize $Z^{\prime}$ is to maximize the 
success rate for matching two subjects whose satisfaction degrees to each other are closed enough.

(M2) can be solved by using the Lagrange function-based method. Let

$$
\Theta\left(\theta_{i j}, \lambda\right)=\sum_{i=1}^{m} \sum_{j=1}^{n} \frac{\theta_{i j}}{e^{\left|a_{i j}-b_{i j}\right|}}+\frac{\lambda}{2}\left(\sum_{i=1}^{m} \sum_{j=1}^{n}\left(\theta_{i j}\right)^{2}-1\right)
$$

where $\lambda$ is the Lagrange parameter.

The partial derivatives pertaining to $\theta_{i j}$ and $\lambda$ are obtained as:

$$
\left\{\begin{array}{l}
\frac{\partial \Theta\left(\theta_{i j}, \lambda\right)}{\partial \theta_{i j}}=\sum_{i=1}^{m} \sum_{j=1}^{n} \frac{1}{e^{\left|a_{i j}-b_{i j}\right|}}+\lambda \theta_{i j} \\
\frac{\partial \Theta\left(\theta_{i j}, \lambda\right)}{\partial \lambda}=\sum_{i=1}^{m} \sum_{j=1}^{n}\left(\theta_{i j}\right)^{2}-1 .
\end{array}\right.
$$

Let $\frac{\partial \Theta\left(\theta_{i j}, \lambda\right)}{\partial \theta_{i j}}=0$ and $\frac{\partial \Theta\left(\theta_{i j}, \lambda\right)}{\partial \lambda}=0$, by solving these two equations, we obtain the normalized form of $\theta_{i j}$, which is shown as:

$$
\theta_{i j}=\frac{1}{\left(\sum_{i=1}^{m} \sum_{j=1}^{n} \frac{1}{e^{\left|a_{i j}-b_{i j}\right|}}\right) e^{\left|a_{i j}-b_{i j}\right|}}
$$

Considering the weights of sides $A$ and $B,(M 1)$ can be transformed to single-objective model $(M 3)$.

$$
(M 2)\left\{\begin{aligned}
& \max Z=\varpi^{B} \sum_{i=1}^{m} \sum_{j=1}^{n} \theta_{i j} a_{i j} x_{i j}+\varpi^{A} \sum_{i=1}^{m} \sum_{j=1}^{n} \theta_{i j} b_{i j} x_{i j}, \\
& \text { s.t. } \quad \sum_{j=1}^{n} x_{i j}=1, i=1,2, \mathrm{~L}, m, \\
& \sum_{i=1}^{m} x_{i j} \leq 1, j=1,2, \mathrm{~L}, n,
\end{aligned}\right.
$$

where the goal function in equation (30) depicts the maximum of the comprehensive satisfaction degree, $\varpi^{A}$ and $\varpi^{B}$ are the weights of sides $A$ and $B$ respectively and satisfy $0 \leq \varpi^{A}, \varpi^{B} \leq 1$, and $\varpi^{A}+\varpi^{B}=1$. (M2) can be demonstrated in two aspects: the matching aspirations and the satisfaction degrees. It is obvious that equation (30) aims to reach the maximum of the matching aspirations, however, an issue remains. From equation (29) we find two closed 
mutual satisfaction degrees make the matching aspiration larger, in other words, the matching aspirations are obtained in the light of the difference between two satisfaction degrees rather than the values of them. If we only consider the matching aspirations and make them to reach their maximum, then two subjects may be matched whose mutual satisfaction degrees are closed but lower than others, which is irrational. If we only consider the satisfaction degrees, although we can reach the maximum of the comprehensive satisfaction degrees, the satisfaction degrees of two matched subjects may be far apart. Although the summation of two mutual satisfaction degrees of two matched subjects is larger, the matching aspiration between this two subjects may be lower, which indicates the lower success rate for matching them. (M2) simultaneously considers the matching aspirations and the satisfaction degrees and effectively overcomes the above mentioned issues. Equation (30) integrates the matching aspirations and the satisfaction degrees by the multiplicative operator. The multiplicative operator can be seen as a restriction, it makes the comprehensive satisfaction degrees reach their maximum and ensures the satisfaction degrees of two matched subjects close enough.

\subsection{Decision Steps}

The decision process can be summarized as the following steps.

Step 1: Obtain the probabilistic linguistic evaluations and build evaluation matrices $E^{1}=\left[e_{\mu, i j}^{1}\right]_{(m \times r) \times n}$ and $E^{2}=\left[e_{v, i j}^{2}\right]_{m \times(n \times t)}$.

Step 2: Normalize the evaluations and transform the evaluations under the cost criteria to the benefit types by utilizing equation (2).

Step 3: Classify the criterion $C_{\mu}^{1}$ and calculate the preference degree of $B_{j}$ to $A_{i}$ compared with other subjects of side $A$ under $C_{\mu}^{1}$ by utilizing equations (3)-(8). Classify the criterion $C_{v}^{2}$ and calculate the preference degree of $A_{i}$ to $B_{j}$ compared with other subjects of side $B$ under $C_{v}^{2}$ by utilizing equations (9)-(14).

Step 4: Aggregate the preference degrees to the preference index for sides $B$ and $A$ by utilizing equations (15) and (16) respectively.

Step 5: Calculate the outgoing flows for sides $B$ and $A$ by utilizing equations (17) and (18) 
respectively. Calculate the incoming flows for sides $B$ and $A$ by utilizing equations (19) and (20) respectively.

Step 6: Calculate the satisfaction degrees of $B_{j}$ to $A_{i}$ and $A_{i}$ to $B_{j}$ by utilizing equations (21) and (22) respectively.

Step 7: Build multi-objectives TSMDM model $(M 1)$.

Step 8: Build model $(M 2)$. By solving $(M 2)$, the values of $\theta_{i j}$ can be obtained shown as equation (29).

Step 9: Transform the multi-objectives TSMDM model $(M 1)$ to the single-objective TSMDM model $(M 3)$.

Step 10: Solve (M3) to obtain the matching scheme.

\section{An Illustrative Example}

Intelligent technology intermediary is a matching intermediary, the main business of which is to polymerize the intelligent technology suppliers and the demanders according to their requirements and mutual evaluations. The intelligent technology intermediary can help the intelligent technology suppliers and the demanders to reduce their cost for searching each other and market survey. The intelligent technology suppliers are individuals or companies who own the knowledge or property for the technology, while the demanders are enterprises who are seeking the technology. There are two distinguished advantages of the intelligent technology intermediary. One is that it is third part and do not participate into the matching process, the recommendation of which is objective. The other is that it gathers the information with respect to the intelligent technology and assesses the suppliers and the demanders previously, the suggestion of which is valuable. In a period, an intelligent technology firm in Fuzhou has received the requests from three demanders and four suppliers, the demanders look for face recognition technology while the suppliers tend to find partners. Owing to the financial pressure, a demander can only purchase the technology from one supplier. It is necessary to avoid the technology monopoly, hence, a supplier can only collaborate with one demander. The demanders are $A_{1}, A_{2}, A_{3}$, and the suppliers are $B_{1}, B_{2}$, 
$B_{3}, B_{4}$. The criteria associated with side $A$ are income $\left(C_{1}^{1}\right)$, scale of enterprise $\left(C_{2}^{1}\right)$, and developing prospect $\left(C_{3}^{1}\right)$, while the criteria associated with side $B$ are research ability $\left(C_{1}^{2}\right)$, value of achievements $\left(C_{2}^{2}\right)$, and fund level $\left(C_{3}^{2}\right)$. After the market survey, the weight vectors of $C^{1}$ and $C^{2}$ are set as $\omega^{1}=(0.4,0.3,0.3)^{\mathrm{T}}$ and $\omega^{2}=(0.5,0.2,0.3)^{\mathrm{T}}$ respectively. $C_{1}^{1}$, $C_{2}^{1}$ and $C_{1}^{2}$ are classified into the type of criteria with linear preference, where $\beta^{1}=0.05$ and $\beta^{2}=0.01$. Other criteria are classified into the type of usual criteria. The basic LTS is $S=\left\{s_{-3}, s_{-2}, s_{-1}, s_{0}, s_{1}, s_{2}, s_{3}\right\}$ which is $\{$ none,very low,low,medium,high,very high,perfect $\}$. The decision steps of the proposed approach are expressed as follows:

Step 1: Obtain the probabilistic linguistic evaluations. The evaluation matrix $E^{1}=\left[e_{\mu, i j}^{1}\right]_{(m \times r) \times n}$ is shown as Table 1 . The evaluation matrix $E^{2}=\left[e_{v, i j}^{2}\right]_{m \times(n \times t)}$ is broke up into Table 2-Table 4 for convenience.

Table 1. Evaluations of side $A$ given by side $B$

\begin{tabular}{|c|c|c|c|c|c|}
\hline & & $B_{1}$ & $B_{2}$ & $B_{3}$ & $B_{4}$ \\
\hline \multirow{3}{*}{$C_{1}^{1}$} & $A_{1}$ & $\left\{s_{2}(0.2), s_{3}(0.8)\right\}$ & $\left\{s_{1}(0.9), s_{2}(0.1)\right\}$ & $\left\{s_{1}(0.5), s_{2}(0.5)\right\}$ & $\left\{s_{0}(0.2), s_{2}(0.8)\right\}$ \\
\hline & $A_{2}$ & $\left\{s_{-1}(0.2), s_{2}(0.8)\right\}$ & $\left\{s_{-1}(0.1), s_{1}(0.9)\right\}$ & $\left\{s_{0}(0.2), s_{1}(0.8)\right\}$ & $\left\{s_{3}(0.9), s_{1}(0.1)\right\}$ \\
\hline & $A_{3}$ & $\left\{s_{-1}(0.5), s_{2}(0.5)\right\}$ & $\left\{s_{1}(0.8), s_{2}(0.2)\right\}$ & $\left\{s_{1}(0.7), s_{3}(0.3)\right\}$ & $\left\{s_{2}(0.1), s_{1}(0.9)\right\}$ \\
\hline \multirow{3}{*}{$C_{2}^{1}$} & $A_{1}$ & $\left\{s_{2}(0.3), s_{1}(0.7)\right\}$ & $\left\{s_{3}(0.1), s_{1}(0.9)\right\}$ & $\left\{\begin{array}{l}s_{0}(0.2), s_{1}(0.2), \\
s_{0}(0.6)\end{array}\right\}$ & $\left\{s_{1}(0.5), s_{2}(0.5)\right\}$ \\
\hline & $A_{2}$ & $\left\{s_{-2}(0.1), s_{1}(0.9)\right\}$ & $\left\{s_{0}(0.5), s_{1}(0.5)\right\}$ & $\left\{s_{-1}(0.9), s_{2}(0.1)\right\}$ & $\left\{s_{1}(0.8), s_{2}(0.2)\right\}$ \\
\hline & $A_{3}$ & $\left\{s_{-1}(0.5), s_{2}(0.5)\right\}$ & $\left\{s_{-2}(0.1), s_{1}(0.9)\right\}$ & $\left\{s_{1}(0.8), s_{2}(0.2)\right\}$ & $\left\{s_{3}(0.1), s_{2}(0.9)\right\}$ \\
\hline \multirow[b]{2}{*}{$C_{3}^{1}$} & $A_{1}$ & $\left\{s_{0}(0.5), s_{1}(0.5)\right\}$ & $\left\{s_{1}(0.3), s_{2}(0.7)\right\}$ & $\left\{s_{1}(0.5), s_{2}(0.5)\right\}$ & $\left\{s_{3}(0.1), s_{1}(0.9)\right\}$ \\
\hline & $A_{2}$ & $\left\{s_{3}(0.9), s_{1}(0.1)\right\}$ & $\left\{\begin{array}{l}s_{0}(0.1), s_{2}(0.2), \\
s_{3}(0.7)\end{array}\right\}$ & $\left\{s_{2}(0.5), s_{3}(0.5)\right\}$ & $\left\{s_{1}(0.2), s_{2}(0.8)\right\}$ \\
\hline
\end{tabular}




$$
A_{3} \quad\left\{s_{2}(0.5), s_{1}(0.5)\right\} \quad\left\{s_{-1}(0.5), s_{0}(0.5)\right\} \quad\left\{s_{0}(0.6), s_{2}(0.4)\right\} \quad\left\{s_{3}(0.1), s_{1}(0.9)\right\}
$$

Table 2. Evaluations of side $B$ given by side $A$ under criterion $C_{1}^{2}$

\begin{tabular}{ccccc}
\hline & $B_{1}$ & $B_{2}$ & $B_{3}$ & $B_{4}$ \\
\hline$A_{1}$ & $\left\{s_{-1}(0.2), s_{0}(0.8)\right\}$ & $\left\{s_{-2}(0.3), s_{-1}(0.7)\right\}$ & $\left\{\begin{array}{l}s_{-1}(0.2), s_{0}(0.5), \\
s_{1}(0.3)\end{array}\right\}$ & $\left\{s_{2}(0.5), s_{0}(0.5)\right\}$ \\
$A_{2}$ & $\left\{s_{2}(0.2), s_{0}(0.8)\right\}$ & $\left\{s_{3}(0.1), s_{0}(0.9)\right\}$ & $\left\{s_{0}(0.8), s_{1}(0.2)\right\}$ & $\left\{s_{0}(1)\right\}$ \\
$A_{3}$ & $\left\{s_{1}(0.8), s_{2}(0.2)\right\}$ & $\left\{s_{0}(0.9), s_{1}(0.1)\right\}$ & $\left\{s_{-2}(0.2), s_{-1}(0.8)\right\}$ & $\left\{s_{-1}(0.5), s_{1}(0.5)\right\}$ \\
\hline
\end{tabular}

Table 3. Evaluations of side $B$ given by side $A$ under criterion $C_{2}^{2}$

\begin{tabular}{ccccc}
\hline & $B_{1}$ & $B_{2}$ & $B_{3}$ & $B_{4}$ \\
\hline$A_{1}$ & $\left\{s_{0}(0.8), s_{1}(0.2)\right\}$ & $\left\{s_{0}(0.7), s_{2}(0.3)\right\}$ & $\left\{s_{0}(0.2), s_{2}(0.8)\right\}$ & $\left\{s_{-2}(0.3), s_{2}(0.7)\right\}$ \\
$A_{2}$ & $\left\{s_{3}(0.1), s_{-1}(0.9)\right\}$ & $\left\{s_{0}(0.6), s_{3}(0.4)\right\}$ & $\left\{s_{-3}(0.1), s_{1}(0.9)\right\}$ & $\left\{s_{-1}(0.5), s_{1}(0.5)\right\}$ \\
$A_{3}$ & $\left\{s_{2}(0.2), s_{1}(0.8)\right\}$ & $\left\{s_{-1}(0.8), s_{0}(0.2)\right\}$ & $\left\{s_{-1}(0.9), s_{1}(0.1)\right\}$ & $\left\{s_{0}(0.7), s_{1}(0.3)\right\}$ \\
\hline
\end{tabular}

Table 4. Evaluations of side $B$ given by side $A$ under criterion $C_{3}^{2}$

$\left.\begin{array}{ccccc}\hline & B_{1} & B_{2} & B_{3} & B_{4} \\ \hline A_{1} & \left\{s_{-1}(0.7), s_{0}(0.3)\right\} & \left\{s_{2}(0.5), s_{1}(0.5)\right\} & \left\{s_{-1}(0.3), s_{1}(0.7)\right\} & \left\{s_{-1}(0.8), s_{0}(0.2)\right\} \\ A_{2} & \left\{s_{-2}(0.1), s_{-1}(0.9)\right\} & \left\{s_{-1}(0.8), s_{2}(0.1)\right\} & \left\{s_{3}(0.1)\right.\end{array}\right\}$

Step 2: The evaluations in Table 1-Tbale 4 are normalized to the form in Table 5-Tbale 8 respectively. The evaluations are transformed by using equation (2). Since the criteria are benefit, the evaluations are not changed. 
Table 5. Evaluations of side $A$ given by side $B$

\begin{tabular}{|c|c|c|c|c|c|}
\hline & & $B_{1}$ & $B_{2}$ & $B_{3}$ & $B_{4}$ \\
\hline \multirow{3}{*}{$C_{1}^{1}$} & $A_{1}$ & $\left\{s_{3}(0.8), s_{2}(0.2)\right\}$ & $\left\{s_{1}(0.9), s_{2}(0.1)\right\}$ & $\left\{s_{2}(0.5), s_{1}(0.5)\right\}$ & $\left\{s_{2}(0.8), s_{0}(0.2)\right\}$ \\
\hline & $A_{2}$ & $\left\{s_{2}(0.8), s_{-1}(0.2)\right\}$ & $\left\{s_{1}(0.9), s_{-1}(0.1)\right\}$ & $\left\{s_{1}(0.8), s_{0}(0.2)\right\}$ & $\left\{s_{3}(0.9), s_{1}(0.1)\right\}$ \\
\hline & $A_{3}$ & $\left\{s_{2}(0.5), s_{-1}(0.5)\right\}$ & $\left\{s_{1}(0.8), s_{2}(0.2)\right\}$ & $\left\{s_{3}(0.3), s_{1}(0.7)\right\}$ & $\left\{s_{1}(0.9), s_{2}(0.1)\right\}$ \\
\hline \multirow{3}{*}{$C_{2}^{1}$} & $A_{1}$ & $\left\{s_{1}(0.7), s_{2}(0.3)\right\}$ & $\left\{s_{1}(0.9), s_{3}(0.1)\right\}$ & $\left\{\begin{array}{l}s_{2}(0.6), s_{1}(0.2), \\
s_{0}(0.2)\end{array}\right\}$ & $\left\{s_{2}(0.5), s_{1}(0.5)\right\}$ \\
\hline & $A_{2}$ & $\left\{s_{1}(0.9), s_{-2}(0.1)\right\}$ & $\left\{s_{1}(0.5), s_{0}(0.5)\right\}$ & $\left\{\begin{array}{l}s_{2}(0.1), s_{-1}(0.9), \\
s_{-1}(0)\end{array}\right\}$ & $\left\{s_{1}(0.8), s_{2}(0.2)\right\}$ \\
\hline & $A_{3}$ & $\left\{s_{2}(0.5), s_{-1}(0.5)\right\}$ & $\left\{s_{1}(0.9), s_{-2}(0.1)\right\}$ & $\left\{\begin{array}{l}s_{1}(0.8), s_{2}(0.2), \\
s_{1}(0)\end{array}\right\}$ & $\left\{s_{2}(0.9), s_{3}(0.1)\right\}$ \\
\hline \multirow{3}{*}{$C_{3}^{1}$} & $A_{1}$ & $\left\{s_{1}(0.5), s_{0}(0.5)\right\}$ & $\left\{\begin{array}{l}s_{2}(0.7), s_{1}(0.3), \\
s_{1}(0)\end{array}\right\}$ & $\left\{s_{2}(0.5), s_{1}(0.5)\right\}$ & $\left\{s_{1}(0.9), s_{3}(0.1)\right\}$ \\
\hline & $A_{2}$ & $\left\{s_{3}(0.9), s_{1}(0.1)\right\}$ & $\left\{\begin{array}{l}s_{3}(0.7), s_{2}(0.2), \\
s_{0}(0.1)\end{array}\right\}$ & $\left\{s_{3}(0.5), s_{2}(0.5)\right\}$ & $\left\{s_{2}(0.8), s_{1}(0.2)\right\}$ \\
\hline & $A_{3}$ & $\left\{s_{2}(0.5), s_{1}(0.5)\right\}$ & $\left\{\begin{array}{l}s_{0}(0.5), s_{-1}(0.5), \\
s_{-1}(0)\end{array}\right\}$ & $\left\{s_{2}(0.4), s_{0}(0.6)\right\}$ & $\left\{s_{1}(0.9), s_{3}(0.1)\right\}$ \\
\hline
\end{tabular}

Table 6. Normalized evaluations of side $B$ given by side $A$ under criterion $C_{1}^{2}$

\begin{tabular}{ccccc}
\hline & $B_{1}$ & $B_{2}$ & $B_{3}$ & $B_{4}$ \\
\hline$A_{1}$ & $\left\{\begin{array}{l}s_{0}(0.8), s_{-1}(0.2), \\
s_{-1}(0)\end{array}\right\}$ & $\left\{\begin{array}{l}\left.s_{-2}(0.3), s_{-1}(0.7),\right\} \\
s_{-2}(0)\end{array}\right\}$ & $\left\{\begin{array}{l}s_{1}(0.3), s_{0}(0.5), \\
s_{-1}(0.2)\end{array}\right\}$ & $\left\{\begin{array}{l}s_{2}(0.5), s_{0}(0.5), \\
s_{0}(0)\end{array}\right\}$ \\
$A_{2}$ & $\left\{s_{2}(0.2), s_{0}(0.8)\right\}$ & $\left\{s_{3}(0.1), s_{0}(0.9)\right\}$ & $\left\{s_{1}(0.2), s_{0}(0.8)\right\}$ & $\left\{s_{0}(0.1), s_{0}(0)\right\}$ \\
$A_{3}$ & $\left\{s_{1}(0.8), s_{2}(0.2)\right\}$ & $\left\{s_{1}(0.1), s_{0}(0.9)\right\}$ & $\left\{s_{-2}(0.2), s_{-1}(0.8)\right\}$ & $\left\{s_{1}(0.5), s_{-1}(0.5)\right\}$ \\
\hline
\end{tabular}

Table 7. Normalized evaluations of side $B$ given by side $A$ under criterion $C_{2}^{2}$ 


\begin{tabular}{ccccc}
\hline & $B_{1}$ & $B_{2}$ & $B_{3}$ & $B_{4}$ \\
\hline$A_{1}$ & $\left\{s_{1}(0.2), s_{0}(0.8)\right\}$ & $\left\{s_{2}(0.3), s_{0}(0.7)\right\}$ & $\left\{s_{2}(0.8), s_{0}(0.2)\right\}$ & $\left\{s_{2}(0.7), s_{-2}(0.3)\right\}$ \\
$A_{2}$ & $\left\{s_{3}(0.1), s_{-1}(0.9)\right\}$ & $\left\{s_{3}(0.4), s_{0}(0.6)\right\}$ & $\left\{s_{1}(0.9), s_{-3}(0.1)\right\}$ & $\left\{s_{1}(0.5), s_{-1}(0.5)\right\}$ \\
$A_{3}$ & $\left\{s_{1}(0.8), s_{2}(0.2)\right\}$ & $\left\{s_{0}(0.2), s_{-1}(0.8)\right\}$ & $\left\{s_{1}(0.1), s_{-1}(0.9)\right\}$ & $\left\{s_{1}(0.3), s_{0}(0.7)\right\}$ \\
\hline
\end{tabular}

Table 8. Normalized evaluations of side $B$ given by side $A$ under criterion $C_{3}^{2}$

\begin{tabular}{|c|c|c|c|c|}
\hline & $B_{1}$ & $B_{2}$ & $B_{3}$ & $B_{4}$ \\
\hline$A_{1}$ & $\left\{s_{0}(0.3), s_{-1}(0.7)\right\}$ & $\left\{s_{2}(0.5), s_{1}(0.5)\right\}$ & $\left\{s_{1}(0.7), s_{-1}(0.3)\right\}$ & $\left\{s_{0}(0.2), s_{-1}(0.8)\right\}$ \\
\hline$A_{2}$ & $\left\{\begin{array}{l}s_{-2}(0.1), s_{-1}(0.9), \\
s_{-2}(0)\end{array}\right\}$ & $\left\{\begin{array}{l}s_{3}(0.1), s_{2}(0.1), \\
s_{-1}(0.8)\end{array}\right\}$ & $\left\{\begin{array}{l}s_{1}(0.9), s_{-1}(0.1), \\
s_{-1}(0)\end{array}\right\}$ & $\left\{\begin{array}{l}s_{2}(0.5), s_{1}(0.5), \\
s_{1}(0)\end{array}\right\}$ \\
\hline$A_{3}$ & $\left\{s_{0}(0.9), s_{-3}(0.1)\right\}$ & $\left\{s_{1}(0.9), s_{3}(0.1)\right\}$ & $\left\{s_{1}(0.2), s_{0}(0.8)\right\}$ & $\left\{s_{1}(0.7), s_{0}(0.3)\right\}$ \\
\hline
\end{tabular}

Step 3: Since $C_{1}^{1}, C_{2}^{1}$ and $C_{1}^{2}$ are the criteria with linear preference, $\beta^{1}=0.05$ and $\beta^{2}=0.01$, equation (5) is used to calculate the preference degree of $B_{j}$ to $A_{i}$ under criteria $C_{1}^{1}$ and $C_{2}^{1}$, equation (11) is used to calculate the preference degree of $A_{i}$ to $B_{j}$ under criterion $C_{1}^{2}$. For other criteria, equations (3) and (9) can be used to calculate the preference degrees. We use the calculation of preference degree $f^{11}\left(e_{1,32}^{1}, e_{1,12}^{1}\right)$ as an example. Since the score of $e_{1,32}^{1}$ is $s_{1.2}$ and the score of $e_{1,12}^{1}$ is $s_{1.1}, e_{1,32}^{1}>e_{1,12}^{1}$. However, due to $d\left(e_{1,32}^{1}, e_{1,12}^{1}\right)=0.025<0.05, A_{3}$ is not completely preferred by $B_{2}$ compared to $A_{1}$, then

$$
f^{11}\left(e_{1,32}^{1}, e_{1,12}^{1}\right)=\frac{0.025}{0.5}=0.5
$$

Step 4: Aggregate the preference degrees to the preference indexes by using equations (15) and (16) respectively.

Step 5: The outgoing flows for sides $B$ and $A$ are calculated by using equations (17) and (18) respectively. The incoming flows for side $B$ and $A$ are calculated by using equations (19) 
and (20) respectively.

Step 6: The satisfaction degrees of $A_{i}$ given by $B_{j}$ and $B_{j}$ given by $A_{i}$ are calculated by using equations (21) and (22) respectively. The satisfaction degrees of $A_{i}$ given by $B_{j}$ are shown as Table 9, while the satisfaction degrees of $B_{j}$ given by $A_{i}$ are shown as Table 10.

Table 9. Satisfaction degrees of $A_{i}$ given by $B_{j}$

\begin{tabular}{ccccc}
\hline & $B_{1}$ & $B_{2}$ & $B_{3}$ & $B_{4}$ \\
\hline$A_{1}$ & 0.8000 & 0.5490 & 0.7001 & -0.4501 \\
$A_{2}$ & 0.4999 & -0.5996 & -0.8000 & 0.8501 \\
$A_{3}$ & -1.2999 & 0.0506 & 0.0998 & -0.5000 \\
\hline
\end{tabular}

Table 10. Satisfaction degrees of $B_{j}$ given by $A_{i}$

\begin{tabular}{ccccc}
\hline & $B_{1}$ & $B_{2}$ & $B_{3}$ & $B_{4}$ \\
\hline$A_{1}$ & -1.4000 & -0.8000 & 1.4000 & 0.8000 \\
$A_{2}$ & -0.0835 & 0.7165 & 0 & -0.8000 \\
$A_{3}$ & 1.2000 & 1 & -2.2000 & 0 \\
\hline
\end{tabular}

Step 7: Build multi-objectives TSMDM model $(M 1)$.

Step 8: Build model $(M 2)$. By solving the $(M 2)$, we obtain the values of $\theta_{i j}$ as shown as Table 11 .

Table 11. Matching aspirations

\begin{tabular}{ccccc}
\hline & $B_{1}$ & $B_{2}$ & $B_{3}$ & $B_{4}$ \\
\hline$A_{1}$ & 0.0294 & 0.0685 & 0.1305 & 0.0756 \\
$A_{2}$ & 0.1466 & 0.0708 & 0.1182 & 0.0508
\end{tabular}


Step 9: Transform the $(M 1)$ to $(M 3)$. Since there is no declaration of weights of two sides, we let $\varpi^{A}=\varpi^{B}=0.5$.

Step 10: Solve $(M 3)$. The matching matrix is

$$
\left[\begin{array}{llll}
0 & 0 & 1 & 0 \\
1 & 0 & 0 & 0 \\
0 & 1 & 0 & 0
\end{array}\right],
$$

which means $A_{1}$ matches $B_{3}, A_{2}$ matches $B_{1}, A_{3}$ matches $B_{2}$, the matching pairs are $\left\{\left(A_{1}, B_{3}\right),\left(A_{2}, B_{1}\right),\left(A_{3}, B_{2}\right)\right\}$.

\section{Analyses}

In this section, we conduct sensitivity analysis and comparative analysis for the proposed TSMDM approach.

\subsection{Sensitivity Analysis}

Weights of sides $A$ and $B$ are $\varpi^{A}$ and $\varpi^{B}$ respectively, which are used to measure the importance of two sides. The weights are not always 0.5 because there are several reasons to make the change of the value of weights, such as cost, remuneration, and reputation. The matching scheme may change according to the value of weights. By varying the value of $\varpi^{A}$ and $\varpi^{B}$ from zero to one consistently, we obtain a group of matching schemes associated with the values of goal function shown as Table 12.

Table 12. Matching schemes and values of goal function

\begin{tabular}{ccc}
\hline$\varpi^{A}, \varpi^{B}$ & Matching schemes & Values of $Z$ \\
\hline$\varpi^{A}=0, \varpi^{B}=1$ & $\left\{\left(A_{1}, B_{3}\right),\left(A_{2}, B_{1}\right),\left(A_{3}, B_{2}\right)\right\}$ & 0.1698 \\
$\varpi^{A}=0.1, \varpi^{B}=0.9$ & $\left\{\left(A_{1}, B_{3}\right),\left(A_{2}, B_{1}\right),\left(A_{3}, B_{2}\right)\right\}$ & 0.1801
\end{tabular}




$$
\begin{array}{lll}
\varpi^{A}=0.2, \varpi^{B}=0.8 & \left\{\left(A_{1}, B_{3}\right),\left(A_{2}, B_{1}\right),\left(A_{3}, B_{2}\right)\right\} & 0.1903 \\
\varpi^{A}=0.3, \varpi^{B}=0.7 & \left\{\left(A_{1}, B_{3}\right),\left(A_{2}, B_{1}\right),\left(A_{3}, B_{2}\right)\right\} & 0.2006 \\
\varpi^{A}=0.4, \varpi^{B}=0.6 & \left\{\left(A_{1}, B_{3}\right),\left(A_{2}, B_{1}\right),\left(A_{3}, B_{2}\right)\right\} & 0.2108 \\
\varpi^{A}=0.5, \varpi^{B}=0.5 & \left\{\left(A_{1}, B_{3}\right),\left(A_{2}, B_{1}\right),\left(A_{3}, B_{2}\right)\right\} & 0.2211 \\
\varpi^{A}=0.6, \varpi^{B}=0.4 & \left\{\left(A_{1}, B_{3}\right),\left(A_{2}, B_{1}\right),\left(A_{3}, B_{2}\right)\right\} & 0.2313 \\
\varpi^{A}=0.7, \varpi^{B}=0.3 & \left\{\left(A_{1}, B_{3}\right),\left(A_{2}, B_{1}\right),\left(A_{3}, B_{2}\right)\right\} & 0.2416 \\
\varpi^{A}=0.8, \varpi^{B}=0.2 & \left\{\left(A_{1}, B_{3}\right),\left(A_{2}, B_{1}\right),\left(A_{3}, B_{2}\right)\right\} & 0.2518 \\
\varpi^{A}=0.9, \varpi^{B}=0.1 & \left\{\left(A_{1}, B_{3}\right),\left(A_{2}, B_{1}\right),\left(A_{3}, B_{2}\right)\right\} & 0.2621 \\
\varpi^{A}=1, \varpi^{B}=0 & \left\{\left(A_{1}, B_{3}\right),\left(A_{2}, B_{1}\right),\left(A_{3}, B_{2}\right)\right\} & 0.2724
\end{array}
$$

Since the basic LTS we used is of seven granularity, the probabilistic linguistic evaluations are all finite, then the transformed satisfaction degrees are restricted in a specific finite interval, the matching aspirations can be subsequently obtained as an exact value. It can be found that the matching scheme $\left\{\left(A_{1}, B_{3}\right),\left(A_{2}, B_{1}\right),\left(A_{3}, B_{2}\right)\right\}$ is unchanged along with the change of the value of weights $\varpi^{A}$ and $\varpi^{B}$. No matter how the weights change, the proposed approach is stable enough to ensure that each subject matches the subject from the other side with the largest matching aspiration and the largest satisfaction degree. The typical example is the matching of $A_{3}$ and $B_{2}$. After the matching of $A_{1}$ and $B_{3}, A_{2}$ and $B_{1}$, there remain $A_{3}, B_{2}$, and $B_{4}$ to be matched. Although the matching aspiration between $A_{3}$ and $B_{4}$ is larger than that between $A_{3}$ and $B_{2}$, the summation of $a_{32}$ and $b_{32}$ is far larger than that of $a_{34}$ and $b_{34}$. Hence, to maximize the goal function, $A_{3}$ matches $B_{2}$. Since the matching scheme is always $\left\{\left(A_{1}, B_{3}\right),\left(A_{2}, B_{1}\right),\left(A_{3}, B_{2}\right)\right\}$, we only consider the satisfaction degrees $a_{13}, a_{21}, a_{32}, b_{13}$, $b_{21}$, and $b_{32}$. The summation of $a_{13}, a_{21}$, and $a_{32}$ is 1.2506 , which is larger than that of $b_{13}$, 
$b_{21}$, and $b_{32}$. Hence, along with the ascending of $\varpi^{A}$ and descending of $\varpi^{B}$, value of $Z$ increases.

On the basis of the two-sided matching introduced in Definition 7, three conditions are satisfied, the criteria, the weights of criteria, the probabilistic linguistic evaluations, and the matching aspirations are all considered and integrated into the proposed TSMDM approach. The stability of the proposed approach is verified in the light of the sensitivity analysis.

\subsection{Comparative Analysis}

We compare the proposed approach with the TSMDM method proposed by Yue et al. (2016) in this subsection.

Yue et al. (2016) proposed a TSMDM method with interval-valued intuitionistic fuzzy sets (IVIFSs) considering the matching aspirations, where the evaluations are expressed as IVIFSs. Yue et al. (2016) recognize the scores of the IVIFSs as the satisfaction degrees and further substitute them into the TSMDM models. Hence, we transform the probabilistic linguistic evaluations to the subscripts of their scores for the illustrative example in Section 4. By using the method proposed by Yue et al. (2016), we obtain the matching aspirations shown as Table 13 and the matching scheme $\left\{\left(A_{1}, B_{3}\right),\left(A_{2}, B_{1}\right),\left(A_{3}, B_{2}\right)\right\}$.

Table 13. Matching aspirations of the cited method

\begin{tabular}{ccccc}
\hline & $B_{1}$ & $B_{2}$ & $B_{3}$ & $B_{4}$ \\
\hline$A_{1}$ & 0.0439 & 0.0708 & 0.0915 & 0.0786 \\
$A_{2}$ & 0.0418 & 0.0915 & 0.1268 & 0.0548 \\
$A_{3}$ & 0.1572 & 0.1250 & 0.0436 & 0.0744 \\
\hline
\end{tabular}

The comparative analysis is separated into four parts as follows:

(1) Although this matching scheme is the same as that obtained from the proposed approach, the matching aspirations are different. In Yue et al. (2016)s' method, although $A_{2}$ matches $B_{1}$, the matching aspiration between them is the lowest no matter in the first column or in the second 
row. The lowest matching aspiration for both $A_{2}$ and $B_{1}$ means the lowest success rate between them, which is contradict with the matching scheme. In the proposed TSMDM approach, the matching aspiration between $A_{2}$ and $B_{1}$ is 0.1466 , which is the largest in the first column and second row. Hence, $A_{2}$ matches $B_{1}$ in the matching scheme obtained from the proposed approach is reasonable.

(2) Yue et al. (2016) use the IVIFSs to express the evaluations. However, the IVIFSs can only describe the quantitative evaluations rather than the qualitative ones. The PLTSs we introduced can describe the qualitative evaluations given by the subjects, which are more convenient.

(3) Yue et al. (2016) straightforwardly transform the fuzzy evaluations to their scores, which may cause the loss of information. We compare the probabilistic linguistic evaluations and derive the mutual preference degrees for two subjects. We consider six conditions and further define different preference functions for six types of criteria.

(4) Yue et al. (2016) don't consider the condition of multi-criteria, which is not useful for reallife problems. We furnish multiple criteria for two sides of subjects to help them evaluate each other comprehensively, which is useful.

\section{Conclusion}

TSMDM approaches are widely used in real life. Considering the comparison between the subjects, we propose a TSMDM approach based on the PROMETHEE, where the evaluations are PLTSs. Six preference functions are firstly extended to accommodate the probabilistic linguistic environment. The subtraction in preference functions are substituted by the distance between the PLTSs. The condition of an evaluation is larger than another but their difference is lower than a threshold is separated to two conditions, that is, a probabilistic linguistic evaluation is larger than another but their distance is lower than a threshold. By using the PROMETHEE, the subjects are compared to other subjects from the same side under each criterion. The net-flows, which are redefined as the satisfaction degrees are also obtained. The matching aspirations are proper tools to measure the success rates for matching two subjects, thus they are combined in the multi-objectives TSMDM model. A novel model aiming to calculate the values of matching aspirations is built, and 
is further solved by using the Lagrange function. We introduce the weights of two sides and further transform the multi-objectives TSMDM model to the single-objective TSMDM model with the derived value of matching aspirations. The proposed TSMDM approach is employed to resolve a matching problem for an intelligent technology intermediary in Fuzhou to verify the feasibility and the effectiveness. The sensitivity analysis is conducted to test the stability of the proposed approach in eleven conditions where the weights of two sides are varied consistently. The matching scheme is unchanged no matter how the weights vary, hence, the proposed TSMDM approach is stable. The comparative analysis between the proposed TSMDM approach and the existing TSMDM method considering the matching aspirations shows the progressiveness in four parts: the value of matching aspirations, the expression of evaluations, the transformation of evaluations, and the condition of multi-criteria.

In the future, we will consider several more complicated conditions for defining the preference functions under the probabilistic linguistic environment. Also, we will investigate the weight determining method for the TSMDM approaches.

Acknowledgments The work was supported by the National Natural Science Foundation of China (No. 61773123).

\section{Compliance with ethical standards}

Conflict of interest The authors declare that they have no known competing financial interests or personal relationships that could have appeared to influence the work reported in this paper.

Ethical standard This article does not contain any studies with human participants or animals performed by any of the author.

\section{References}

Brans JP, Vincke Ph (1985) A preference ranking organisation method: (The PROMETHEE method 
for multiple criteria decision-making). Management Science 31: 647-656

Chen CT, Hung WZ, Cheng HL (2011) Applying linguistic PROMETHEE method in investment portfolio decision-making. International Journal of Electronic Business Management 9: 139148

Dağdeviren M (2008) Decision making in equipment selection: an integrated approach with AHP and PROMETHEE. Journal of Intelligent Manufacturing 19: 397-406

Fan ZP, Li MY, Zhang X (2017) Satisfied two-sided matching: a method considering elation and disappointment of agents. Soft Computing 22: 7227-7241

Gale D, Shapley LS (1962) College admissions and the stability of marriage. American Mathematical Monthly 69: 9-15

Gou XJ, Xu ZS (2016) Novel basic operational laws for linguistic terms, hesitant fuzzy linguistic term sets and probabilistic linguistic term sets. Information Sciences 372: 407-427

Han J, Li B, Liang HM, Lai KK (2018) A novel two-sided matching decision method for technological knowledge supplier and demander considering the network collaboration effect. Soft Computing 22: 5439-5451

Kobina A, Liang DC, He X (2017) Probabilistic linguistic power aggregation operators for multicriteria group decision making. Symmetry 9: 320

Liang DC, He X, Xu ZS (2020) Multi-attribute dynamic two-sided matching method of talent sharing market in incomplete preference ordinal environment. Applied Soft Computing 93: 106427

Liang R, Wu CZ, Sheng ZH, Wang XY (2018) Multi-criterion two-sided matching of public-private partnership infrastructure projects: criteria and methods. Sustainability 10: 1178-1200

Liao HC, Jiang LS, lev B, Fujita H (2019) Novel operations of PLTSs based on the disparity degrees of linguistic terms and their use in designing the probabilistic linguistic ELECTRE III method. Applied Soft Computing 80: 450-464

Liao HC, Jiang LS, Xu ZS, Xu JP, Herrera F (2017) A linear programming method for multiple criteria decision making with probabilistic linguistic information. Information Sciences 415416: $341-355$

Lin MW, Xu ZS (2017) Probabilistic linguistic distance measures and their applications in multicriteria group decision making. Soft Computing Applications for Group Decision-Making and 
Consensus Modeling 357: 411-440

Lin Y, Wang YM, Chin KS (2018) An enhanced approach for two-sided matching with 2-tuple linguistic multi-attribute preference. Soft Computing 23: 7977-7990

Liu HB, Jiang L, Xu ZS (2018) Entropy measures of probabilistic linguistic term sets. International Journal of Computational Intelligence Systems 11: 45-57

Liu PD, Li Y (2018) The PROMETHEE II method based on probabilistic linguistic information and their application to decision making. Informatica 29: 303-320

Liu Y, Li KW (2017) A two-sided matching decision method for supply and demand of technological knowledge. Journal of Knowledge Management 21: 592-606

Pang Q, Wang H, Xu ZS (2016) Probabilistic linguistic term sets in multi-attribute group decision making. Information Sciences 369: 128-143

Rodriguez RM, Martinez L, Herrera F (2012) Hesitant fuzzy linguistic term sets for decision making. IEEE Transactions on Fuzzy Systems 20: 109-119

Vinodh S, Girubha RJ (2012) PROMETHEE based sustainable concept selection. Applied Mathematical Modelling 36: 5301-5308

Vujosevic ML, Popovic MJ (2016) The comparison of the energy performance of hotel buildings using PROMETHEE decision-making method. Thermal Science 20: 197-208

Wang J, Li B, Li W, Yang SL (2018) Two-sided matching model based on cumulative prospect theory for decision making of two-way referral system. Journal of Interdisciplinary Mathematics 21: 1097-1102

Wang PY, Shen J, Zhang B (2016) A new method for two-sided matching decision making of PPP projects based on intuitionistic fuzzy choquet integral. Journal of Intelligent \& Fuzzy Systems 31: $2221-2230$

Wang XK, Wang JQ, Zhang HY (2019) Distance-based multicriteria group decision-making approach with probabilistic linguistic term sets. Expert Systems 36: 1-18

Wu XL, Liao HC, Xu ZS, Hafezalkotob A, Herrera F (2018) Probabilistic linguistic MULTIMOORA: A multicriteria decision making method based on the probabilistic linguistic expectation function and the improved Borda rule. IEEE Transactions on Fuzzy Systems 26: $3688-3702$

Xu ZS (2012) Linguistic decision making: Theory and method. Berlin: Springer 
Yin S, Li BZ (2018) Matching management of supply and demand of green building technologies based on a novel matching method with intuitionistic fuzzy sets. Journal of Cleaner Production 201: 748-763

Yu DJ, Xu ZS (2019) Intuitionistic fuzzy two-sided matching model and its application to personnelposition matching problems. Journal of the Operational Research Society 71: 312-321

Yu XB, Chen H, Ji ZH (2019) Combination of probabilistic linguistic term sets and PROMETHEE to evaluate meteorological disaster risk: Case study of southeastern China. Sustainability 11: 1405

Yue Q, Zhang L, Peng YS, Yu BW, Hong Y, Xiao Q (2016) Decision method for two-sided matching with interval-valued intuitionistic fuzzy sets considering matching aspirations. Journal of Intelligent \& Fuzzy Systems 31: 2903-2910

Yue Q, Zhang LL (2020) Two-sided matching for hesitant fuzzy numbers in smart intelligent technique transfer. Mechanical Systems and Signal Processing 139: 106643

Yue Q, Zhang LL, Yu BW, Zhang LJ, Zhang JL (2019) Two-sided matching for triangular intuitionistic fuzzy numbers in smart environmental protection. IEEE Access 7: 42426-42435

Zadeh LA (1975) The concept of a linguistic variable and its application to approximate reasoningI. Information Sciences 8: 199-249 